Klaus TAUSEND - Gabriele ERATH

\title{
DIE FUNDSTELLE LAKKOMATA IM GRENZGEBIET VON PHENEOS, ORCHOMENOS UND KAPHYAI*
}

Einer der bedeutendsten Verkehrswege der antiken Peloponnes ${ }^{1}$ verband die arkadischen Poleis Orchomenos und Pheneos ${ }^{2}$ und stellte einen der Hauptanmarschwege nach Norden für die Truppen Spartas dar ${ }^{3}$. Die Schlüsselstelle dieses bereits von Pausanias ${ }^{4}$ ausführlich beschriebenen Weges befindet sich am höchsten Punkt der Route, wo die von Orchomenos kommende Straße eine kleine Hochebene verläßt und durch die vom Saitasgebirge (antik: Oryxis) im Westen und dem Skipieza (antik: Skiathis) im Osten gebildete Schlucht in das Becken von Pheneos herabführt, das sie beim antiken Ort Karyai nördlich des heutigen Dorfes Mati erreicht. Hier trafen auch nach Pausanias die Grenzen der antiken Polisterritorien von Pheneos, Orchomenos und Kaphyai aufeinander, und an eben dieser Stelle zweigte ein antiker Weg von der Hauptstraße Pheneos-Orchomenos nach Westen ab und führte nach Kaphyai. Ebenso wie die OrchomenosPheneos-Straße ist auch der Weg nach Kaphyai sowohl durch die Beschreibung bei Pausanias als auch durch archäologische Überreste wie Trassierung und Wagenspuren belegt (Abb. 1).

Abgesehen von der unzweifelhaft vorhandenen verkehrstechnischen Bedeutung dieser Stelle erbrachte eine Begehung des Geländes zusätzliche bemerkenswerte Ergebnisse. Zuvor jedoch scheint eine kurze topographische Beschreibung des Gebietes angebracht ${ }^{5}$.

Verläßt man - von Norden kommend - die genannte Schlucht zwischen Saitas und Skipieza (antik: Oligyrtos), so gelangt man zunächst auf eine kleine dreieckige Hochfläche (ca. $300 \mathrm{~m} \times$ $250 \mathrm{~m} \times 500 \mathrm{~m}$ ), welche etwa $1050 \mathrm{~m}$ hoch gelegen von mehreren niedrigen Hügeln (bis zu $30 \mathrm{~m}$ ) unterbrochen wird. Begrenzt wird diese Hochfläche im Norden durch die Abhänge des $1695 \mathrm{~m}$ hohen Gremos, eines Teils des Saitas, im Nordosten und Osten durch das Massiv des Oligyrtos und im Süden durch den 1149 m hohen Prophitis Ilias. Nach Süden setzt sich diese Ebene in einer breiten Senke zwischen Oligyrtos und Prophitis Ilias fort, durch welche der Weg nach Orchomenos verläuft. Im Westen trennt eine schmale Engstelle zwischen Gremos und Prophitis Ilias diese Hochebene von einer zweiten, die sich ca. $600 \mathrm{~m}$ am Abhang des Gremos entlangzieht und sich etwa 250 m nach Süden erstreckt. Dieses ebenfalls von mehreren Hügeln bestandene Gebiet wird aufgrund der flachen Senken zwischen den einzelnen Hügeln Lakkomata genannt. Am Nordrand dieser Hochebene verläuft der antike Weg nach Kaphyai (Abb. 2).

\footnotetext{
* Die folgenden Ausführungen sind gleichsam als 'Nebenprodukt' eines vom FWF geförderten Forschungsprojektes zur historischen Topographie von Pheneos entstanden, dessen Ergebnisse in K. Tausend (Hrsg.), Pheneos und Lousoi. Untersuchungen zur Geschichte und Topographie Nordostarkadiens (1999) publiziert wurden. An dieser Stelle sei den Mitarbeiter(inne)n des ÖAI Athen, vor allem Frau Doz. V. Mitsopoulos-Leon, sowie den Damen und Herren der Ephorie Nafplion für ihre Unterstützung bei allen unseren Forschungsprojekten im Raum Pheneos herzlichst gedankt.

1 Während diese Straßenverbindung noch im vorigen Jahrhundert bestand und häufig begangen wurde, existieren heute in diesem Gebiet lediglich Feldwege.

2 Zu diesem Weg s. K. Tausend, Der antike Weg von Pheneos nach Orchomenos, ÖJh 67, 1998, 109-116.

3 Dies gilt vor allem für die Zeit des Peloponnesischen Krieges (s. Thuk. 2, 9, 2; 5, 57-60), aber auch für die Auseinandersetzungen zwischen Sparta und dem Achäischen Bund im 3. Jh. v. Chr. (Polyb. 4, 13, 4).

${ }^{4}$ Paus. 8, 13, 4-6.

5 Zur Geologie des Gebietes sei A. Philippson, Die griechischen Landschaften III. Der Peloponnes (1959) 237 zitiert: »Hier taucht der Tripolitsa-Kalk nach S bald unter stark gefältelten Olonoskalk hinab, der kahle und öde Plateaus von 1000-1200 m ü. M. bildet, die sich gegen die Ebene von Kandila erstrecken.«
} 


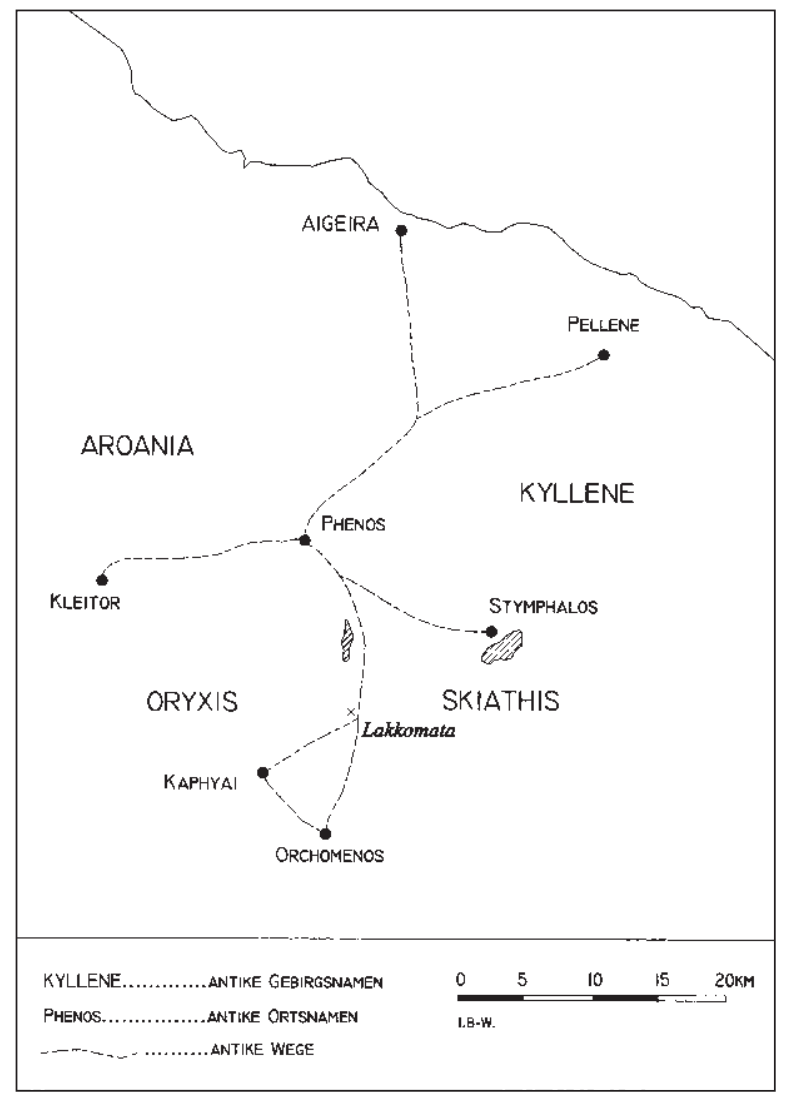

1 Nordostarkadien im Altertum
Im Zuge eines vom Fonds zur Förderung der wissenschaftlichen Forschung geförderten Surveys des Polisterritoriums von Pheneos konnten nun auf beiden beschriebenen Hochebenen, die ja nur durch eine Engstelle getrennt sind, archäologische Funde gemacht werden.

Auf der erwähnten dreieckigen Hochfläche befinden sich auf dem nördlichsten der kleinen Hügel, unmittelbar südlich der Stelle, an der die antiken Straßen von Orchomenos, Kaphyai und Pheneos aufeinandertreffen, die spärlichen Überreste eines antiken Bauwerkes. Die Kuppe dieses felsigen Hügels wurde abgearbeitet, so da $\beta$ eine ebene Fläche von ca. 5-6 m im Quadrat entstand, die offensichtlich als Fundament für einen Bau diente. Etwas unterhalb dieser abgearbeiteten Fläche, am westlichen Abhang des Hügels, befinden sich vier behauene Quader bzw. deren Bruchstücke, die wohl Bestandteil des Bauwerkes gewesen sein dürften und herabgestürzt waren. Sowohl auf der abgearbeiteten Hügelkuppe selbst als auch am Westabhang nahe der Steinquader finden sich auch Bruchstücke antiker Keramik, die in spätklassische Zeit zu datieren sind. Zusam-
5. oder Anfang des 4. Jahrhunderts v. Chr. menfassend kann man daher feststellen, daß Ende des 5. oder Anfang des 4. Jahrhunderts v. Chr.
auf der Kuppe dieses Hügels ein kleines Gebäude - wohl ein Wachturm - stand, dessen primäre Funktion die Überwachung dieses verkehrstechnisch so wichtigen Punktes an der Route PheneosOrchomenos-Kaphyai war.

Am Fuße dieses Hügels wie auch rund um die anderen Erhebungen der kleinen Hochfläche befinden sich zahlreiche rezente Bruchsteinmauern, die allerdings vereinzelt auch antike Blöcke beinhalten; diese Mauerkonstruktionen dienen offensichtlich den lokalen Hirten als Schafshürden. Unter ihnen sticht jedoch eine Konstruktion besonders ins Auge: Es handelt sich hierbei um zwei Mauerstücke, die sowohl im Westen als auch im Osten an den Hügel, der den Turm trug, anschließen (Abb. 3). Die Mauer verlief offenbar über die gesamte kleine Hochfläche und besaß einen Durchlaß für die Trasse der antiken Straße. Zwar ist der Bau an mehreren Stellen durch moderne Feldwege zerstört, doch zeigen die noch erhaltenen Teile - vor allem an der Ostseite des Hügels, wo die Mauer über eine Länge von $10 \mathrm{~m}$ vorhanden ist, - eine auffällige Konstruktionsweise. Der Bau aus Bruchsteinmauerwerk ist bis zu sechs Lagen hoch $(1,20 \mathrm{~m})$ erhalten und besteht aus zwei Schalen im Abstand von ca. 3 m, wobei der Zwischenraum mit Geröll verfüllt ist. Dies unterscheidet sich nun sowohl durch die Schalenbauweise von den übrigen Bruchsteinmauern als auch dadurch, daß das Mauerwerk der Schalen wesentlich fester gefügt ist. Zudem ist der Zweck dieser Mauer - anders als bei den übrigen, leicht als Schafs- und Ziegenställe zu erkennenden Anlagen - nicht zu eruieren. Es liegt daher nahe, daß sie nicht rezenten Ursprunges ist und ehemals einem völlig anderen Zweck diente. Sofern diese Anlage antik ist - Klarheit in dieser Frage könnten nur genauere archäologische Untersuchungen bringen -, handelte es sich dabei wohl um keine fortifikatorische Anlage im engeren Sinne. Als solche hätte sie nämlich nicht ihren Sinn erfüllt, da sie zwar einen Teil der Paßhöhe sperrte, aber einen Durchlaß für den Weg besaß und zudem im Osten wie im Westen leicht zu umgehen war. Möglicherweise handel- 
te es sich bei dieser Mauer, ihren antiken Ursprung immer vorausgesetzt, um eine Art Grenzmarkierung am 'Dreiländereck' PheneosOrchomenos-Kaphyai. Solche Grenzmauern zwischen zwei Polisterritorien existierten des öfteren im antiken Griechenland ${ }^{6}$; das geographisch nächste erhaltene Beispiel einer Grenzmauer befindet sich im Gebiet des heutigen Dorfes Elaiochori bei Tripolis und markierte die antike Grenze zwischen den Territorien von Tegea und Argos ${ }^{7}$.

Festzuhalten bleibt, daß es sich bei den archäologischen Überresten um eine kleine militärische Anlage, wohl um einen Wachturm, gehandelt hat, die eine verkehrstechnische Schlüsselstelle der Route PheneosOrchomenos-Kaphyai kontrollierte. Möglicherweise stand mit diesem Turm auch eine äußerst selten zu findende antike Grenzmarkierungsmauer in Verbindung, welche das Territorium von Pheneos von jenem der Orchomenier und Kaphyaten

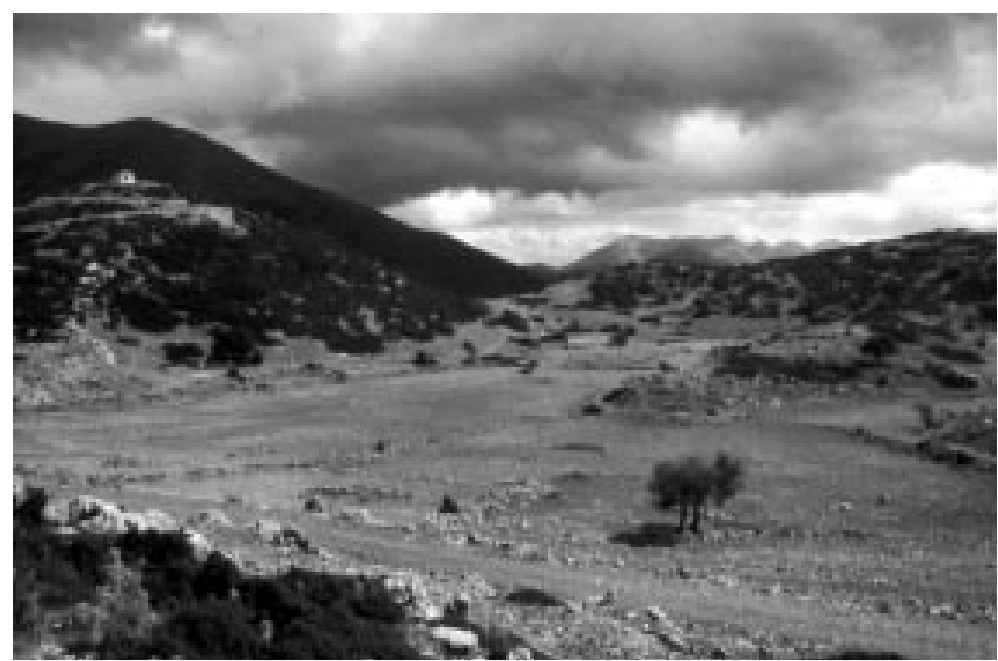

2 Lakkomata von Süden. Im Hintergrund links der Hügel Prophitis Ilias

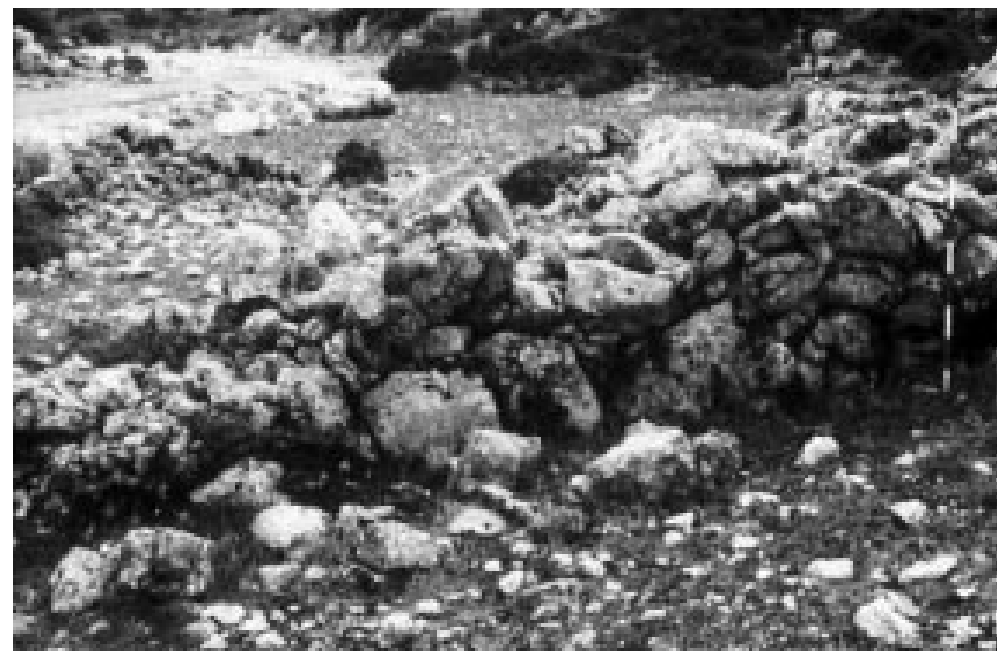

3 Westlicher Teil der 'Grenzmauer' von Lakkomata schied.

Wie schon erwähnt, wird diese Hochfläche durch eine schmale, ca. 100 m lange Engstelle zwischen dem Gremos und dem Prophitis Ilias mit einer zweiten, größeren Hochfläche verbunden. Am Eingang dieser Ebene, unmittelbar vor der Engstelle, befindet sich eine am Abhang des Gremos entspringende, sehr wasserreiche Quelle, die noch heute die gesamte Umgebung versorgt. Das sich anschließende Gebiet - Lakkomata - besteht aus drei unterschiedlichen Geländeformen: aus den zunächst sanft, dann immer steiler ansteigenden Südabhängen des Gremos, aus fünf völlig ebenen, fruchtbaren Senken, die dem Gebiet den Namen gegeben haben, und drei felsigen Hügeln, die sich zwischen den Senken bzw. an ihrem südlichen Rand erheben.

Das gesamte Gebiet kann als geschlossenes archäologisches Fundareal angesprochen werden, wobei die Fundstücke bezüglich ihres Charakters jedoch sehr ungleich auf die unterschiedlichen topographischen Gegebenheiten verteilt sind. Das umfangreichste Fundmaterial tritt

\footnotetext{
s. Strab. 1, 4, 7, der Grenzmarkierungen verschiedener Art aufführt, darunter auch $\pi \varepsilon \rho i ́ \beta o \lambda o t$.

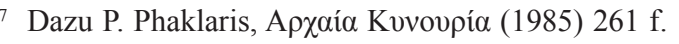




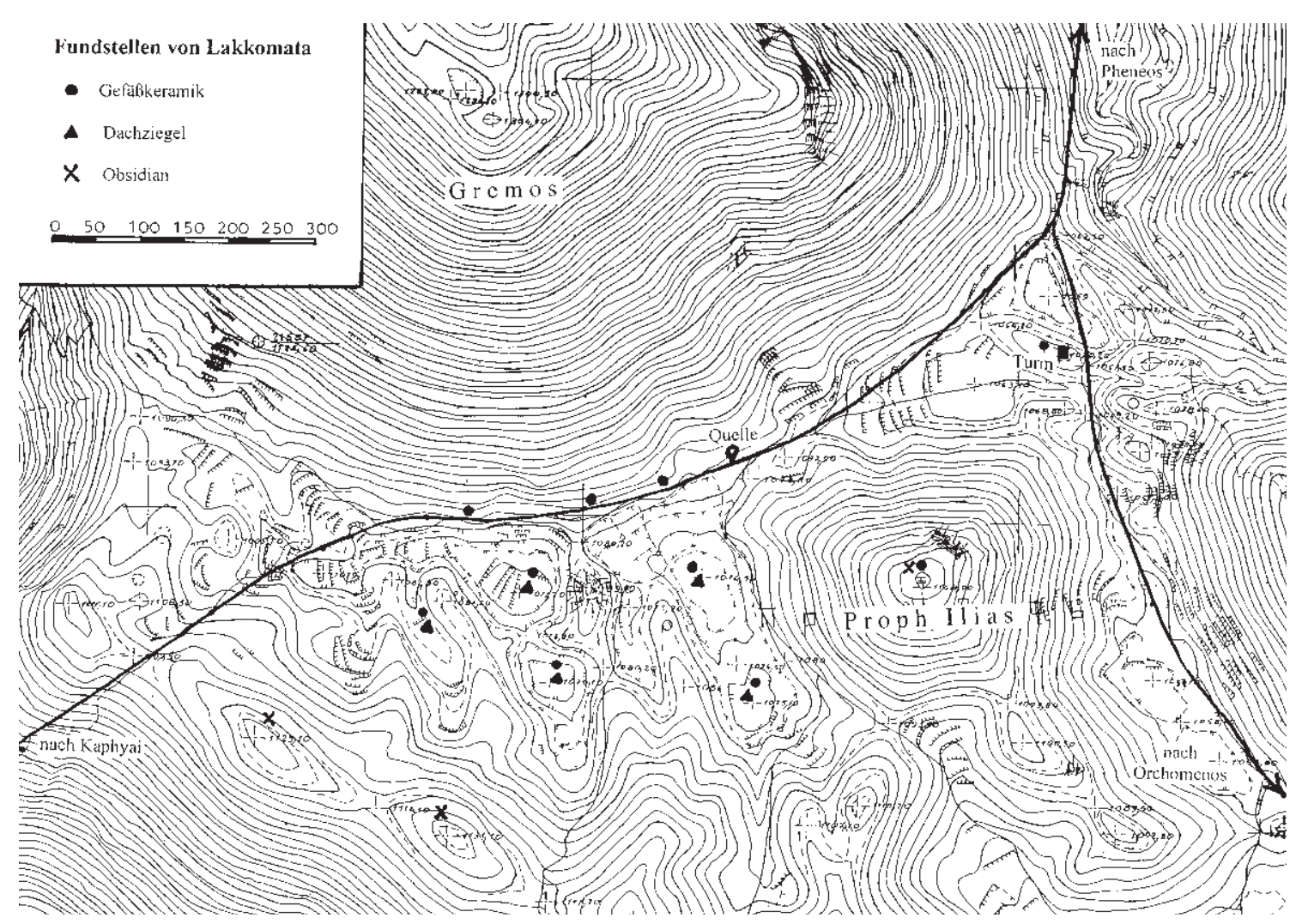

4 Die Fundstellen von Lakkomata

naturgemäß in den Senken beinahe der ganzen Hochfläche zutage, da diese Senken landwirtschaftlich genutzt werden, wobei die Funddichte nach Süden und Westen hin aber deutlich abnimmt. Es handelt sich dabei fast ausschließlich um Tonfragmente, sowohl um Gefäßkeramik als auch - und das in überaus großer Zahl - um Dachziegel. Ganz anders stellt sich die Situation am Nordrand des Fundgebietes, am Abhang des Gremos dar. Hier wurde vor einigen Jahren mit Schubraupen eine Forststraße in den Hang gebaut, die dem Nordrand der Senke entlang nach Westen verläuft. Durch die Bautätigkeit wurde ein 2-3 m breiter Streifen vom Hang weggeschoben, so daß am Nordrand der Straße ein ca. $200 \mathrm{~m}$ langer und bis zu $2 \mathrm{~m}$ hoher Anschnitt entstanden ist. In diesem Profil - und im geringen Ausmaß auf der Forststraße selbst - sind nun ebenfalls zahlreiche Fundstücke vorhanden: hier handelt es sich jedoch vor allem um Reste von Gefäßkeramik, kaum um Dachziegel; hinzu treten noch an zwei Stellen Reste verbrannter Knochen tierischer Provenienz (ca. 0,5 m unter der Erdoberfläche) sowie einige Bruchstücke Obsidian. Auf den drei Felshügeln schließlich, die sich auf der Hochfläche erheben, finden sich - im krassen Gegensatz zu den umliegenden Senken - kaum Keramikreste, jedoch zahlreiche Obsidianfragmente, und zwar sowohl Abschläge als auch Klingen und Schaber.

Zuletzt sei auch noch kurz auf den die gesamte Hochfläche beherrschenden und diese um ca. $80 \mathrm{~m}$ überragenden Hügel eingegangen, auf dessen Spitze sich die Kirche des Agios Ilias befindet. Auf dem Hügel selbst finden sich keinerlei archäologischen Reste, und das Gipfelplateau ist durch den Neubau der Kirche völlig zerstört. Etwa lediglich $10 \mathrm{~m}$ unterhalb des Gipfels, entlang des Zufahrtsweges zur Kirche sind in der durch den Wegbau entstandenen Abrißkante an der Nordseite des Hügels zahlreiche Fundstücke anzutreffen. Es handelt sich wiederum um Obsidianbruchstücke sowie um einige Keramikreste, die allerdings stark ausgeglüht sind. Keramikreste wie auch Obsidian finden sind demnach auch in einer deutlichen, ca. 0,5 m dicken Brandschicht, etwa 1,5 m unter der Erdoberfläche (Abb. 4). 
Bezüglich der chronologischen und typologischen Einordnung der Fundstücke sei auf die Ausführung von G. Erath verwiesen, doch sei an dieser Stelle soviel gesagt, daß im Gegensatz zur archaischen und klassischen Keramik, die sich im gesamten Gebiet findet, prähistorische (mykenische) Keramik bislang primär am westlichen Rand, den Felshügeln und auf dem Prophitis Ilias festgestellt werden konnte.

Bei dem Fundgebiet von Lakkomata handelt es sich wohl um einen Siedlungsplatz - worauf vor allem die große Zahl Dachziegelreste schließen läßt -, der vor allem in archaischer und klassischer Zeit bewohnt war. Er befand sich in einer verkehrstechnisch vorteilhaften Lage am Kreuzungspunkt drei wichtiger antiker Straßen Nordostarkadiens und konnte somit, zumal die fortifikatorische Anlage an der Wegkreuzung wohl nicht von der Siedlung zu trennen ist, diese Schlüsselstelle kontrollieren. Die Siedlung war zudem durch eine ergiebige Quelle ausreichend mit Wasser versorgt und verfügte mit dem Hügel Prophitis Ilias über eine kleine, natürlich befestigte Akropolis. Was das anbaufähige Ackerland betrifft, war die Siedlung jedoch geländebedingt sehr eingeschränkt, da nur die wenigen kleinen Senken von Lakkomata für den Getreideanbau überhaupt in Frage kommen. Es ist daher anzunehmen, daß für die Bewohner von Lakkomata auch im Altertum die Kleintierhaltung die wirtschaftliche Grundlage bildete.

Da diese Siedlung genau am 'Dreiländereck' der Territorien von Pheneos, Orchomenos und Kaphyai liegt, ist schwer zu entscheiden, zu welcher Polis sie politisch gehörte ${ }^{8}$. Die geographisch nächstgelegene Stadt ist zwar Kaphyai, doch führt der kürzeste Weg von Lakkomata zur pheneatischen Kome Karyai (beim heutigen Mati), so daß eine Zugehörigkeit von Lakkomata zu Pheneos wahrscheinlicher ist. Auch ist der Wachturm an der Wegkreuzung so angelegt, daß man zwar eine weite Strecke in Richtung Orchomenos und Kaphyai einsehen konnte, ein aus Pheneos anrückendes Heer jedoch erst wenige hundert Meter vor Erreichen der Hochfläche zu sehen gewesen wäre, weshalb der Turm höchstwahrscheinlich zum Verteidigungssystem von Pheneos gehört haben wird.

Über die Dauer der Besiedelung von Lakkomata läßt sich ohne intensivere Untersuchungen nichts Näheres sagen. Es ist allerdings auffällig, daß Pausanias, der die Route von Orchomenos nach Pheneos bereist hat und neben der ausführlichen Wegbeschreibung auch das 'Dreiländereck' erwähnt, kein Wort über diese Siedlung verliert. Zwar liegt sie eigentlich an der Straße nach Kaphyai, die Pausanias offenbar nicht begangen hat, doch befindet sie sich so nahe an der vom Periegeten ausführlich besprochenen Kreuzung, daß sie unmöglich der Aufmerksamkeit eines Reisenden entgangen sein konnte. Und handelte es sich bei Lakkomata auch nur um eine kleine Ansiedlung, so erwähnt Pausanias doch die Komen Karyai ${ }^{9}$ und Lykouria ${ }^{10}$ im Territorium von Pheneos, da sie an seinem Reiseweg lagen. Schließlich verleiht auch die topographische Lage im Grenzgebiet dreier Poleis der Siedlung von Lakkomata einige Besonderheit, so daß man eine Erwähnung erwarten dürfte. Es liegt daher der Schluß nahe, daß zu Pausanias' Zeit an dieser Stelle keine Siedlung mehr bestand hat und auch keine erwähnenswerten archäologischen Reste mehr vorhanden waren. In diesem Fall müssen die geringfügigen Reste römischer Keramik (s.u.) anders erklärt werden ${ }^{11}$.

Was schließlich die prähistorische Besiedelung dieses Platzes betrifft, so ist der Befund noch zu dürftig, um daraus Schlüsse zu ziehen. Lediglich das häufige Auftreten von Obsidian sowie die Brandschicht am Prophitis Ilias deuten darauf hin, daß auch in mykenischer Zeit hier ein

\footnotetext{
${ }^{8}$ Eine politische Selbständigkeit der Siedlung von Lakkomata wird man allenfalls für die früharchaische Zeit in Erwägung ziehen dürfen, da in späteren Quellen nur die Orte Orchomenos, Pheneos und Kaphyai als Poleis dieses Gebietes genannt werden.

9 Paus. 8, 13, 4 .

10 Paus. $8,19,4$.

11 Das Spektrum reicht hier wohl von der Existenz einer römischen Villa bis zu einem Streufund ohne Aussagekraft. Es besteht allerdings auch die Möglichkeit, daß die Siedlung zur Zeit des Pausanias zwar existierte, der Perieget sie aber nicht erwähnte. Es sei nur daran erinnert, daß er für das Stadtgebiet von Pheneos, in dem er sich selbst aufhielt, das einzige archäologisch belegbare Heiligtum, jenes des Asklepios, nicht erwähnt.
} 
häufig besuchter Ort existierte. Ob es sich dabei aber um ein kleines Höhenheiligtum, eine Siedlung oder einen militärischen Wachposten - vergleichbar jenem auf dem Prophitis Ilias oberhalb von Mykene ${ }^{12}$ - handelte, muß vorerst offen bleiben.

Prof. Dr. Klaus Tausend

Institut für Alte Geschichte und Altertumskunde, Karl-Franzens-Universität Graz, Universitätsplatz 3/II, A-8010 Graz

E-Mail: sabine.tausend@kfuni.graz.ac.at

\section{Fundmaterial aus der Flur Lakkomata}

Im Zuge der in der Flur Lakkomata durchgeführten Begehungen wurden drei bis dahin in der archäologischen Fachwelt unbekannte Fundstellen genauer untersucht ${ }^{13}$. Die auffälligste und flächenmäßig ausgedehnteste Fundstelle liegt in einer Senke westlich des Prophitis Ilias und zieht sich bis über einen Weg, der dieses Gebiet in Ost-West-Richtung erschließt, auf den Hangfuß. Eine weitere Fundstelle ist auf einer Geländewelle unweit des südlichen Endes der Schlucht von Mati situiert. Auf dem Berg Prophitis Ilias schließlich, der die Senke vom Hauptteil der Hochfläche abtrennt, konnte eine dritte Fundstelle beobachtet werden.

Die erste Fundstelle erbrachte, bedingt durch ihre Großflächigkeit, die größte Anzahl an Fundstücken. Das Fundmaterial besteht zum überwiegenden Teil aus Gefäßkeramik sowie einer Einsatzhilfe für einen Töpferofen und Baukeramik in Form von Dachziegeln, wodurch der Fundplatz mit einiger Wahrscheinlichkeit als Siedlungsstelle angesprochen werden kann.

Die Gefäßkeramik setzt sich aus mehreren Warengruppen zusammen, von denen chronologisch eine kleine Gruppe von drei Fragmenten mykenischer Keramik den Beginn markiert. Der Scherben aller drei Fragmente zeichnet sich durch einen grauen Kern und einen beigefarbigen Überzug aus. Das Wandfragment einer Amphora mit glänzend schwarzem Spiral- oder Argonautendekor (Abb. 5) besitzt einige chronologische Empfindlichkeit und findet Vergleiche in der Phase SH IIIA $1^{14}$. Das Fußfragment einer Kylix ${ }^{15}$ weist sowohl außen als auch im Schalenboden Reste eines roten bis schwarzbraunen Überzuges auf (Abb. 6).

Eine relativ einheitliche Warengruppe bilden Fragmente korinthischer Keramik. Das charakteristische Fabrikat besitzt einen hellockerfarbigen Ton mit grünlichem oder rötlichem Einschlag und mit feiner, kaum sichtbarer Magerung, die in einigen Fällen allerdings etwas gröber und von weißer Farbe sein kann. Die Oberfläche ist durch Verwitterungsprozesse stark mitgenommen, so daß sich von der ehemaligen Bemalung oft nur die negative Evidenz dort feststellen läßt, wo die Bemalung abgesprungen ist und das Dekor sich durch den Kontrast des hellen Tons von der verschmutzten Oberfläche gut abhebt. An Fragmenten mit guterhaltener Oberfläche läßt sich die Firnisfarbe als schwarzbraun beschreiben, darunter liegt in einigen Fällen ein hellbrauner Überzug. Das Formenspektrum umfaßt mit Pyxiden, Skyphosschalen und Kotylen Gefäße kleineren Formats, wird aber durch wenige Fragmente größerer Gefäße wie einer Kanne ${ }^{16}$ und eines offenen Gefäßes (Abb. 12) ${ }^{17}$ abgerundet.

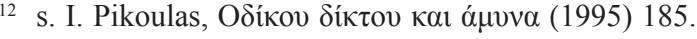

13 Die Funde der ersten beiden Stellen wurden jüngst publiziert in: G. Erath, Archäologische Funde im Becken von Pheneos, in: Tausend (Anm. *) 228-235 Taf. 14, 3-16, 4 (im Folgenden Erath, Funde).

14 Erath, Funde 229 K1 Taf. 14, 3; vgl. P. A. Mountjoy, Mycenaean Decorated Pottery (1986) 43 Abb. 47, 5.

15 Erath, Funde 229 K2 Taf. 14, 6; vgl. C. W. Blegen, Zygouries (1928) 151-153 Nr. 73 Taf. 118; Tiryns. Forschungen und Berichte V (1971) 67 Nr. 20 Taf. 36.

16 Bandhenkel einer Kanne: Erath, Funde 230 K9.

17 Vielleicht eine Lekane oder ein Krater: Dm der Mündung ca. 25-30 cm.
} 

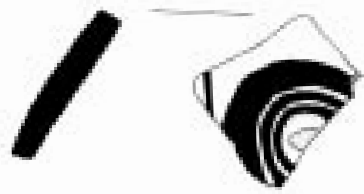

5 Lakkomata. Amphora SH III A1, Wandfrgt.

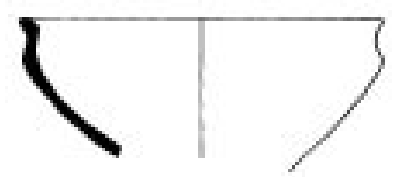

7 Lakkomata. Korinthische Skyphosschale, Frgt.
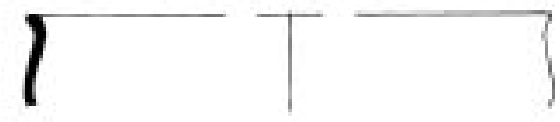

9 Lakkomata. Korinthische Skyphosschale, Randfrgt.

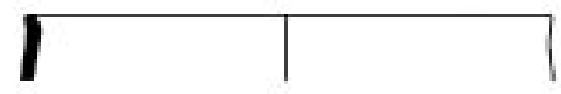

10 Lakkomata. Korinthischer Skyphos

mit Wulstrand, Randfrgt.

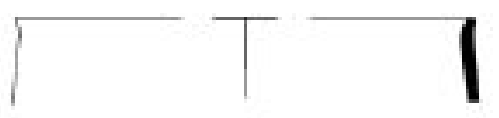

6 Lakkomata. Mykenische Kylix, Fußfrgt.

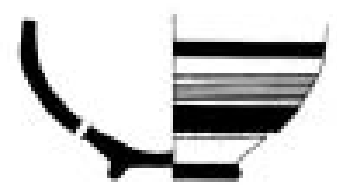

8 Lakkomata. Korinthische Kugelpyxis, Frgte.

\footnotetext{
11 Lakkomata. Korinthischer Skyphos mit Wulstrand, Randfrgt.
} 


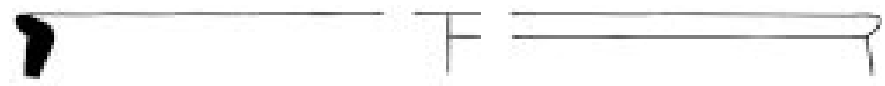

12 Lakkomata. Korinthisches Gefäß (Lekane/Krater), Randfrgt.

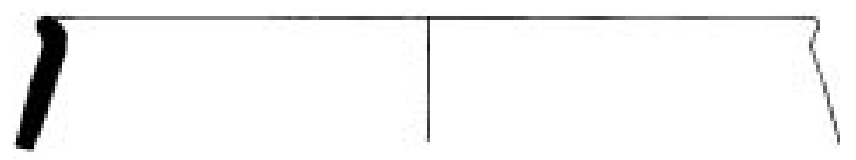

13 Lakkomata. Topf mit Wulstrand, Randfrgt.

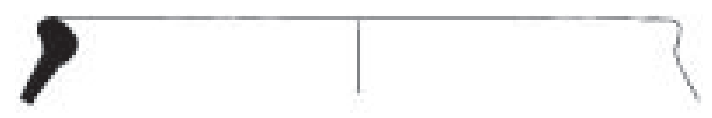

14 Lakkomata. Chytra, Randfrgt.

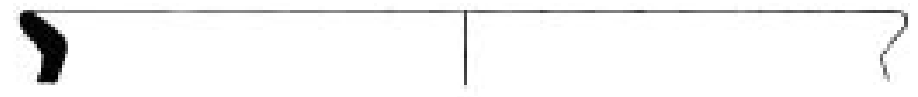

15 Lakkomata. Schüssel, Randfrgt.

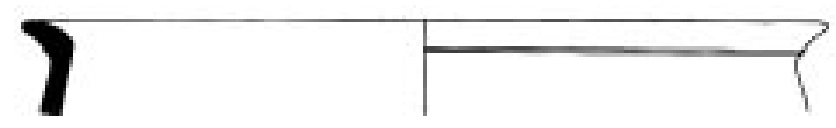

16 Lakkomata. Topf, Randfrgt.

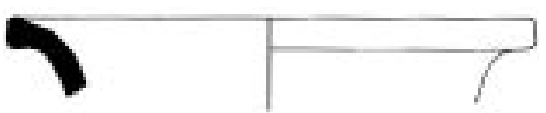

17 Lakkomata. Kanne mit verdicktem Rand, Randfrgt.
18 Lakkomata. Gefäß mit horizontal ausgestellter Lippe (Hydriskos/ Schale), Randfrgt. 


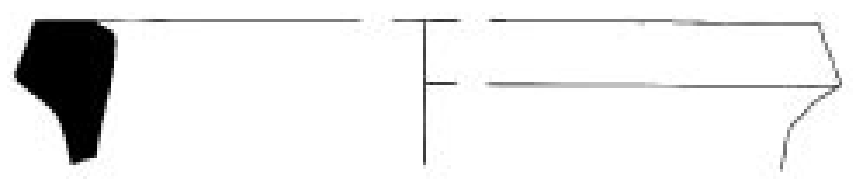

19 Lakkomata. Pithos mit kantiger Lippe, Randfrgt.

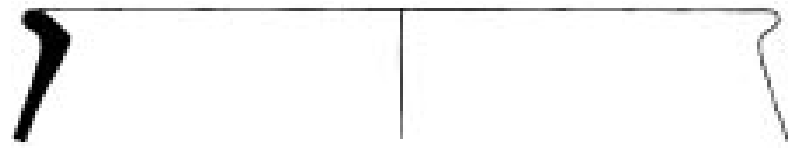

20 Lakkomata. Topf, Randfrgt.

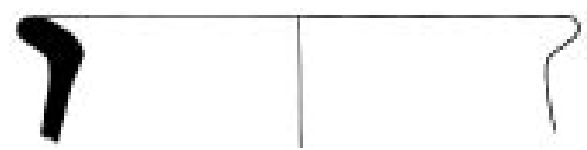

21 Lakkomata. Topf, Randfrgt.

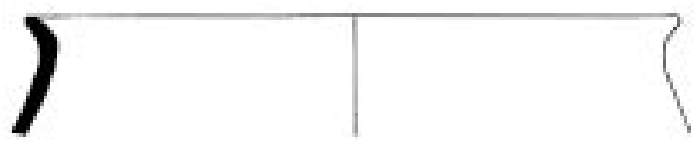

22 Lakkomata. Topf, Randfrgt.
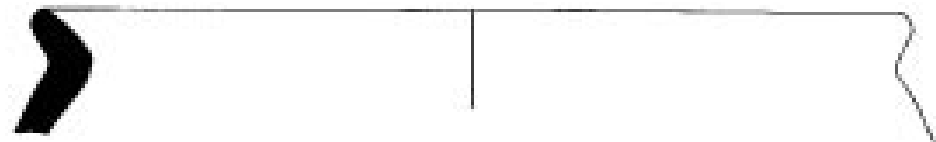

23 Lakkomata. Topf, Randfrgt. 


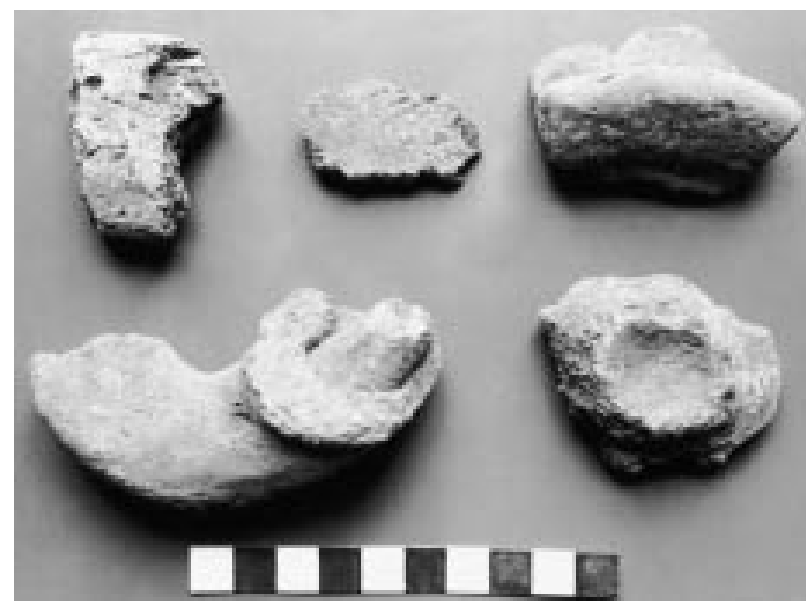

24 Lakkomata. Grobkeramik aus der Senke (Fundstelle 1)

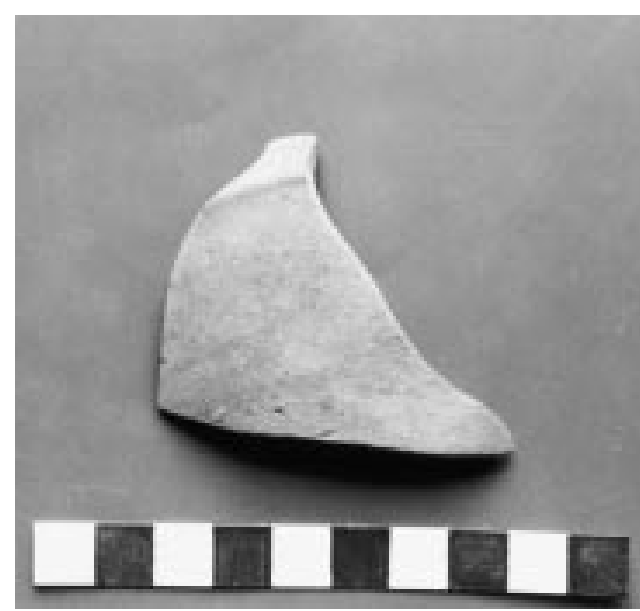

25 Lakkomata. Grautonige Keramik aus der Senke (Fundstelle 1)

Die Fragmente von Kotylen mit Streifenbemalung und undekorierten Skyphosschalen überwiegen bei weitem. Die Skyphosschale Abbildung 7 zeichnet sich durch eine Kehlung des Randes und durch einen knapp unterhalb der Mündung befindlichen Knick der Gefäßwand aus ${ }^{18}$. Der Schalenkörper zieht unter dem Knick stark ein. An der Skyphosschale (Abb. 9) wird der Wandungsknick tiefer und sanfter durchgeführt ${ }^{19}$. Steilwandige Skyphoi mit Wulstrand (Abb. 10-11) ${ }^{20}$ finden sich wie Skyphosschalen sowohl im korinthischen Repertoire als auch in der elischen und messenischen Keramik. Zwei Fragmente einer Kugelpyxis (Abb. 8) ${ }^{21}$ sowie ein fragmentierter Pyxidendeckel sind Vertreter einer Gefäßgattung, die man nicht unbedingt in einem Siedlungskontext vermuten würde ${ }^{22}$. Der Wulstrand eines Topfes aus porösem, ockerfarbenen Ton mit dunkler Magerung sei den korinthischen Stücken als möglicherweise heimisches Erzeugnis nachgestellt (Abb. 13) ${ }^{23}$.

Eine dritte Warengruppe setzt sich aus rot- und orangetoniger Keramik zusammen. Firnis in schwarzbrauner, glänzender Qualität ist nur selten nachzuweisen ${ }^{24}$, der größte Teil der Keramik ist ohne Überzug oder Firnis auf uns gekommen. Die Gruppe umfaßt die jüngst publizierten Stücke sowie die Neufunde aus der Begehung im Jahre 1999. Schwierigkeiten in der exakten

${ }_{18}$ Dm der Mündung $11 \mathrm{~cm}$; Scherben grünlichocker: Erath, Funde 230 K4 Taf. 14, 4. Für dieses Stück lassen sich Vergleiche aus dem späten 6. Jh. anführen: S. S. Weinberg, The Geometric and Orientalizing Pottery, Corinth VII 1 (1943) 69 Nr. 212. 213. 287; vgl. A. Newhall Stillwell - J. L. Benson, The Potters' Quarter. The Pottery, Corinth XV 3 (1984) Nr. 1494.

19 Dm nicht bestimmbar. Vgl. J. Schilbach, Elische Keramik des 5. und 4. Jahrhunderts, OF 23 (1995) Taf. 30, 2 (470/55); Taf. 30, 3 (465/55). Schilbach a. O. 42 betont den Einfluß attischer Skyphosschalen auf die Formgebung der olympischen Gefäße. Tatsächlich wäre auch bei Abb. 9 attischer Einfluß zu überlegen, eine Datierung in das 2. Viertel des 5. Jhs. scheint durchaus gerechtfertigt.

${ }^{20} \mathrm{Dm} 16 \mathrm{~cm}$ (Abb. 10) bzw. nicht bestimmbar (Abb. 11). Als Vergleiche können olympische Gefäße angeführt werden: W. Gauer, Die Tongefäße aus den Brunnen unterm Stadion-Nordwall und im Südost-Gebiet, OF 8 (1975) Abb. 23, 7. 8 (für letztes Exemplar wird eine korinthische Werkstatt vermutet). Gauer datiert beide Stücke nach der Mitte des 5. Jhs.; vgl. auch den spätarchaisch datierten Komplex von Kopanaki in Messenien: N. Kaltsas, AEphem 1983, 207-237 Abb. 9, 3014.

${ }_{21}$ Dm des Standringes 4 cm, Scherben grünlichocker, Bemalung mattbraun: Erath, Funde 230 K5. K6; vgl. J. Boardman - J. Hayes, Excavations at Tocra 1963-1965. The Archaic Deposits I (1966) Nr. 156 und für das Dekor ebenda Nr. 149 (beides spätkorinthisch).

22 The Laconia Survey. Continuity and Change in a Greek Rural Landscape II (1996) 87.

${ }_{23}$ Dm der Mündung ca. 24 cm: Erath, Funde 231 f. K13; vgl. Gauer (Anm. 20) 84 Abb. 3,15 (spätarchaisch). Die Scherbenbeschreibung würde gut zum pheneatischen Fragment passen.

${ }^{24}$ Wandfrgt.: Erath, Funde 231 K12. 


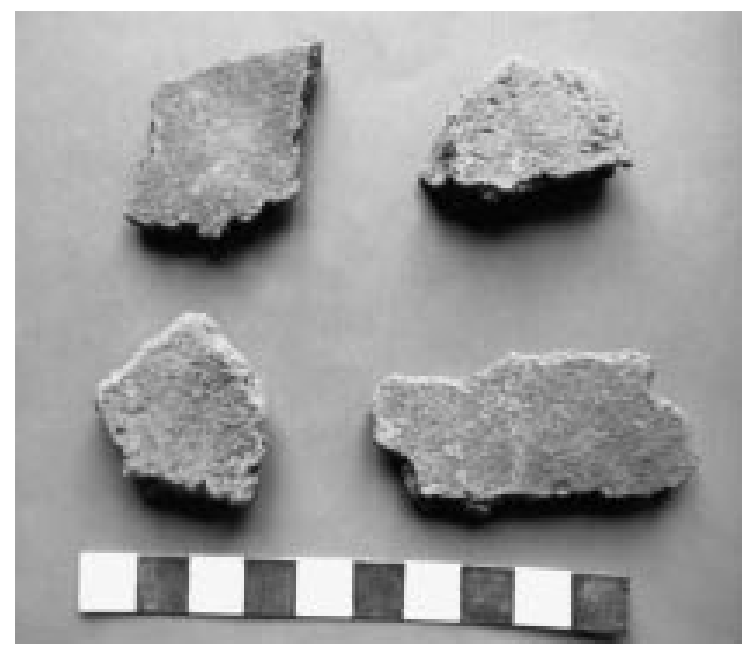

26 Grobkeramik vom Prophitis Ilias (Fundstelle 3)

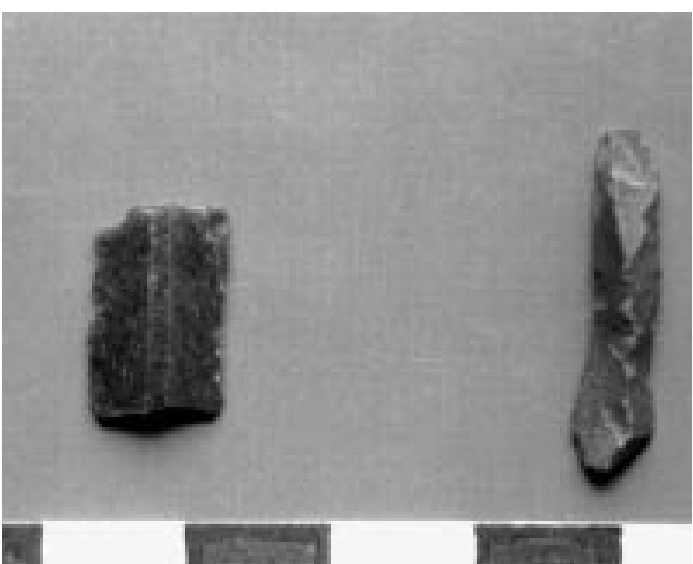

27 Obsidianklinge und Silex vom Prophitis Ilias (Fundstelle 3)

Ansprache der Gefäßtypen und vor allem in der chronologischen Bestimmung sind darin begründet, daß der arkadischen Gebrauchsware in der Keramikforschung bisher wenig Aufmerksamkeit zuteil wurde, daher Vergleichsmaterial auf breiter und chronologisch abgesicherter Basis weitgehend fehlt. Das Formenspektrum wird von Schalen bzw. Skyphoi ${ }^{25}$ dominiert, daneben sind Formen nachzuweisen, die wahrscheinlich der Speisenzubereitung gedient haben, so etwa eine Chytra (Abb. 14) ${ }^{26}$ und eine Schüssel (Abb. 15) 27 $^{27}$ die in ihrem Randprofil durchaus Ähnlichkeit mit einem Fragment rottoniger, hartgebrannter Ware aus Lakkomata besitzt (Abb. 16) ${ }^{28}$. An geschlossenen Gefäßen sind eine Kanne mit verdicktem Rand (Abb. 17) ${ }^{29}$ und ein Gefäß mit horizontal ausgestellter, dünnwandiger Lippe (Abb. 18) ${ }^{30} \mathrm{zu}$ erwähnen. Das Randfragment eines schweren Pithos mit kantig geformter Lippe (Abb. 19) ${ }^{31}$ findet archaische bis klassische Parallelen in Messenien und Lakonien ${ }^{32}$. Die herangezogenen Vergleiche legen eine Datierung der Gruppe in archaische bis klassische Zeit nahe.

Eine weitere größere Gruppe bilden rot- und grautonige Waren. Merkmale dieser Ware sind der klirrend hart gebrannte Scherben und nach außen gebogene Ränder, die innen mit einem

${ }^{25}$ Erath, Funde 231 Nr. K11 Taf. 14, 7; vgl. Thorikos 1970/71. Rapport préliminaire sur les septième et huitième campagnes de fouilles (1978) Nr. 81 Abb. 42 (archaisch); J. E. Coleman, Excavations at Pylos in Elis (1986) 40 ff. Abb. 7; Gauer (Anm. 20) 151 ff. Abb. 15, 5 (6. Jh.). Es sei angemerkt, daß die Form von Coleman als typisch elisch bezeichnet wird.

26 Dm der Mündung 19 cm; vgl. Agora XII Nr. 1932 Abb. 18 (330-305 v. Chr.).

27 Dm der Mündung ca. 27 cm; vgl. F. Lang, Archaische Siedlungen in Griechenland (1996) 84 Abb. 17, 7, die eine attische Lekane als Vergleich anführt. Ausschwingende Wandungen würde man aber eher bei Töpfen vermuten.

${ }^{28}$ Erath, Funde 232 K16. Ein Vergleich dieses Randstückes mit einer in der Kehlung unter dem Rand sitzenden Leiste ist wieder in Babes zu finden: Lang (Anm. 27) 84 Abb. 17, 9 (schlägt 5. Jh. vor), obwohl der Rand des pheneatischen Stückes nicht hakenförmig nach unten gezogen ist.

29 Erath, Funde 232 f. K20. Dazu vgl. J. Boardman - J. Hayes, Excavations at Tocra 1963-1965. The Archaic Deposits II and Later Deposits (1973) 39 f. Nr. 2110 (2. Viertel 6. Jh.) und Gauer (Anm. 20) Abb. 2, 2 (2. Viertel 6. Jh. mit einer Laufzeit des Profils bis an das Ende der Archaik).

${ }^{30}$ Aufgrund der geringen Wandstärke der Lippe kann das Gefäß nicht allzu große Dimensionen besessen haben. Die Lippenform spräche für einen Hydriskos oder eine Flasche: vgl. in etwa Lang (Anm. 27) 88 Abb. 19, 10. Sie schlägt mit einiger Vorsicht ein hellenistisches Datum vor. Als weiteres, wenn auch örtlich weit entferntes Beispiel sei eine Bauchlekythos aus Kampanien erwähnt: A. D. Trendall, Red figure vases of South Italy and Sicily (1989) Abb. 294 (Foundling painter, ca. Mitte 4. Jh. v. Chr.).

31 Dm der Mündung $31 \mathrm{~cm}$ : Erath, Funde $233 \mathrm{~K} 21$ Taf. 16, 3.

32 Kaltsas (Anm. 20) 223 f. 235 ff. Abb. 12, am ehesten Nr. 52 (archaisch); Laconia Survey (Anm. 22) Abb. 14. 17. $21(600-300)$. 
scharfen Knick in die Gefäßwand umbiegen. An der Innenseite sind Drehrillen sichtbar. Im Formenspektrum befinden sich vor allem Gefäße mit weiter Mündung ${ }^{33}$, deren Gefäßwand nach außen ausschwingt und die mit einiger Vorsicht derzeit nur allgemein als Töpfe zu klassifizieren sind (Abb. 20-23). Ähnliche Randbildungen sind auch an Fragmenten mit weicherem Scherben zu beobachten. Durch Vergleiche mit olympischen und messenischen Stücken könnte vielleicht für diese Gruppe eine Datierung in das 6. Jahrhundert und die erste Hälfte des 5. Jahrhunderts vorgeschlagen werden. Die ungewöhnliche Härte des Scherbens wird auch für die frühklassische Keramik Olympias vermerkt ${ }^{34}$.

Als Gruppe ist auch eine Ware mit sehr grober, dunkelbrauner Textur und orangebrauner Oberfläche zu nennen (Abb. 24). Vertreter dieser Ware sind ein horizontaler Henkel mit einem Zapfen zum Einsetzen in die Gefäßwand, ein Bodenfragment und ein Randfragment mit gerade abschließender Lippe. Die Ware ist derzeit chronologisch nicht näher einzuordnen.

Fragmente mit grauem Scherben, der fein gemagert und hart gebrannt ist und weder Überzug noch Bemalung aufweist, können als eine kleine Gruppe zusammengestellt werden (Abb. 25). Ein Wandfragment mit scharf profiliertem Wandungsknick hat vielleicht zu einer Schale gehört ${ }^{35}$. Mit aller Vorsicht soll eine hellenistische Datierung ins Auge gefaßt werden.

Als chronologischer 'Ausreißer' figuriert ein glattes Wandfragment einer italischen oder südgallischen Sigillata ${ }^{36}$. Römisch-kaiserzeitliche Keramik ist bis auf ein weiteres, fragliches Fragment ${ }^{37}$ von der Fundstelle bis dato nicht bekannt.

An der zweiten, auf einer Geländewelle nahe beim südlichen Ausgang der Schlucht von Mati gelegenen Fundstelle wurde schließlich ein Mauerzug aus Bruchsteinen beobachtet (s. o.), in dessen Umgebung sich Fragmente wahrscheinlich klassischer Schwarzfirnisware nachweisen ließen. Schwarzfirnisware ist eine bei Wachtürmen klassischen oder hellenistischen Datums häufig anzutreffende Keramikgattung ${ }^{38}$.

An der dritten, auf dem Gipfel des Prophitis Ilias entdeckten Fundstelle konnten mehrere Fragmente grober Ware mit orangebrauner Oberfläche und an ihr sichtbarer Magerung beobachtet werden (Abb. 26). Als neue Fundkategorie für den Fundplatz sind Steinartefakte in Form einer Obsidianklinge ${ }^{39}$ und eines rotbraunen Flintes ${ }^{40}$ zu vermerken (Abb. 27).

Mit diesem kurzen Überblick läßt sich zeigen, daß das Fundmaterial Belege für menschliche Anwesenheit schon ab der Bronzezeit (mykenische Keramik in der Senke, Obsidiane und Silex auf dem Prophitis Ilias) liefert. Mykenische Funde im Becken von Pheneos wurden auf der Akropolis von Pheneos und an der im nördlichen Teil des Beckens gelegenen Fundstelle Agios Charalambos bekannt ${ }^{41}$. Ein retuschierter Silex wurde außerdem auf dem nördlich des antiken

33 Abb. 20: Dm der Mündung 23 cm: Erath, Funde 231 f. K14 Taf. 15, 2; Abb. 21: Dm der Mündung 17 cm: Erath, Funde 232 K15 Taf. 15, 3; Abb. 22: Dm der Mündung 20 cm: Erath, Funde 232 K17 Taf. 15, 5; Abb. 23: Dm der Mündung $27 \mathrm{~cm}$ : Erath, Funde $232 \mathrm{~K} 18$ Taf. 16, 1.

34 Gauer (Anm. 20) 92.

35 Vgl. etwa Agora XXIX Nr. 1576 und 1577 (beide im Zeitraum von 160-130 v. Chr.).

36 Erath, Funde 233 K22. Freundliche Auskunft von Univ.-Doz. Dr. G. Piccottini.

37 Randfrgt. einer Schüssel mit profilierter Mündung: Erath, Funde 234 K24.

38 Vgl. etwa für das Becken von Pheneos die Fundstellen bei Agios Charalambos (Erath, Funde 214 f.) und Messino (Erath, Funde 217 f.) sowie allgemein J. M. McCredie, Fortified Military Camps in Attica (1966) $99 \mathrm{f}$.

39 Maße: B 9 mm, L 19 mm, D 3 mm; dorsal bilateral retuschiert. Vgl. etwa Agora XIII Taf. 28 Nr. 400: FH-MH(?). Das Stück aus Lakkomata entspricht in seinen Maßen in etwa den Klingen von Agios Charalambos im Norden des Beckens. Dazu s. Laconia Survey (Anm. 22) Abb. 18, 3. 6b. c; J. E. Coleman, Kephala. A Late Neolithic Settlement and Cemetery, Keos I (1977) Nr. 177 Taf. 69.

40 Maße: B 5 mm, L 23 mm, D 4 mm; vielleicht ebenfalls eine Klinge. Vgl. G. C. Nordquist, A Middle Helladic Village. Asine in the Argolid (1987) Kat. 032 Abb. 26 (MH/SH).

${ }^{41}$ Akropolis von Pheneos: G. Erath in: Tausend (Anm. *) 188 f.; E. Protonotariou-Deilaki, ADelt 20, 1965, Chron 158 f.; R. Howell, BSA 65, 1970, Taf. 34a, 10-13; Abb. 5, 11; R. Hope Simpson, A Gazeteer and Atlas of Mycenaean Sites (1965) 37 Nr. 83; ders., Mycenaean Greece (1981) 89 D20 Kalyvia. - Agios Charalambos: Erath, Funde 212. - Erwähnt seien außerdem die südlich in der Ebene von Orchomenos liegenden mykenischen Fundplätze: R. Hope Simpson, Mycenaean Greece (1981) 87 f. und Karte auf S. 86 sowie das südöstlich von Pheneos gelegene antike Stymphalos: Hope Simpson a. O. 89. 
Pheneos gelegenen Hügel Agios Georgios entdeckt ${ }^{42}$. Geräte und Abschläge aus Silex sowie mittelhelladische Keramik sind auch an der Fundstelle Tsoukka am Westrand des Beckens zu belegen ${ }^{43}$. Der größte Teil der Keramik aber stammt aus archaischer und klassischer Zeit und ist an den Fundstellen in der Senke und beim Wachturm nachzuweisen. Durch diesen chronologischen Schwerpunkt wird Lakkomata in das Netz der anderen, im Becken von Pheneos liegenden Fundplätze mit archaischem und klassischem Fundmaterial eingebunden ${ }^{44}$. Vereinzelte Fragmente bringen schließlich Nachweise auch für den Hellenismus und die römische Kaiserzeit.

Die Inhomogenität und die breite zeitliche Streuung des Materials würden eine genauere Untersuchung des Gebietes verlangen. Zu klären wäre etwa die Lage von Wohn- und Wirtschaftsgebäuden, die an den Berghängen zu vermuten sind ${ }^{45}$, während die Senke wahrscheinlich für landwirtschaftliche Zwecke genutzt wurde. Ein intensiver Survey, eine geophysikalische Prospektion oder eventuell sogar eine Grabung könnten die sehr komplexe Situation erhellen.

Mag. Dr. Gabriele Erath

Institut für Archäologie, Karl-Franzens-Universität Graz, Universitätsplatz 3/II, A-8010 Graz

E-Mail: gabriele.erath@kfunigraz.ac.at

Abbildungsnachweis: Abb. 1: Zeichnung I. Benda-Weber; Abb. 2. 3: Photos K. Tausend; Abb. 4: Zeichnung K. Tausend nach Kartengrundlage (1:5000) des Greek Geographical Army Service; Abb. 5-27: Zeichnungen G. Erath.

42 Erath, Funde 217 Taf. 10, 6.

43 Erath, Funde 222 f. Taf. 11.

${ }^{44}$ Die Siedlungsfundstelle Alt-Lykouria in der Flur Roideta mit dem interessanten Fund einer spätarchaischen Ziegelritzinschrift liegt nordwestlich von Lakkomata am Westrand des Beckens: Erath, Funde $223 \mathrm{f}$. Spätarchaische und frühklassische Funde sind außerdem aus den als Heiligtümer zu klassifizierenden Fundstellen der HermesHöhle am Kyllene (Erath, Funde 242 ff.) und des sog. Heiligtums der Artemis Pyronia vom Dourdouvana (Erath, Funde 238 ff.) bekannt geworden. - Erwähnenswert ist weiter der Fund einer spätarchaischen Bronzestatuette eines Hirten in der Flur Triantaphyllia: Erath, Funde 194. 235; N. M. Verdelis, ADelt 19, 1964; M. Jost, Sanctuaires et cultes d'Arcadie (1985) 37. - Nicht ungenannt bleiben soll eine aus Kalavryta stammende Inschrift, die mit dem Kult der Demeter Thesmia in Verbindung gebracht wird: IG V 2, 401; L. H. Jeffery, Local Scripts of Archaic Greece (1961) 209. 214 Nr. 3 Taf. 40 (spätes 6. bzw. 5. Jh.); SEG 11 (1950) Nr. 1112; SEG 22 (1967) Nr. 320; Erath, Funde 239 f. - Ein dem pheneatischen Hermes geweihter Eberkopf wird in der Archäologischen Sammlung Winterthur aufbewahrt: IG V 2, 360; Jeffery a. O. 216 Nr. 34; Jost a. O. 29; Erath, Funde 197; Das Tier in der Antike. Ausstellung Universität Zürich (1974) 28 Kat. 162 Taf. 26 (dort ins späte 5. bis frühe 4. Jh. datiert).

45 Lang (Anm. 27) 55. 
